\title{
Vadim Atsarkin: On the Occasion of His 85th Birthday
}

\author{
Edward B. Fel'dman ${ }^{1}$
}

Published online: 24 May 2021

(c) The Author(s), under exclusive licence to Springer-Verlag GmbH Austria, part of Springer Nature 2021

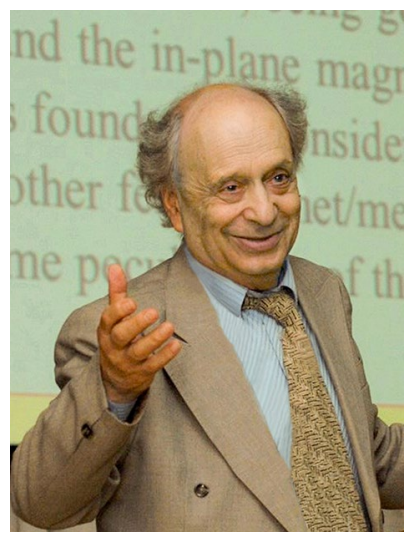

It is a true pleasure to celebrate a friend, an outstanding colleague, and a thinker. Hence, the pleasure I took in editing this Special Issue of Applied Magnetic Resonance dedicated to the 85th birthday of Vadim Aleksandrovich Atsarkin, a renowned leader in the field of magnetic resonance and a friend of many years. He has long served as a Principal Research Scientist at the Kotel'nikov Institute of Radio Engineering and Electronics in Moscow and has led the institute to a distinguished place in the world of magnetic resonance. Professor Atsarkin's fundamental contributions to the development of spin thermodynamics in solid electron and nuclear paramagnets were honored with a Zavoisky Award in 2015.

Vadim Atsarkin was born in Moscow on June 13th, 1936. He graduated with honors from the Physics Department of the Moscow State University in 1959. Soon after his graduation, V. A. Atsarkin started his research at the Quantum Electronics Laboratory headed by Professor Zhabotinskii at the Radio

Edward B. Fel'dman

efeldman@icp.ac.ru

1 Theoretical Department of the Institute of Problems of Chemical Physics of Russian Academy of Sciences, Av. Academician N. N. Semenov, Chernogolovka, 142432 Moscow Region, Russia 
Engineering and Electronics Institute. His first steps in science involved materials for masers with improved parameters. That was of great importance for recently discovered supersensitive quantum amplifiers. The research method was intimately connected with Electron Paramagnetic Resonance (EPR) discovered by E. K. Zavoisky in 1944 at the Kazan State University. In the field that was completely new to him, V. A. Atsarkin quickly achieved significant progress and successfully defended his candidate of science (Ph.D.) thesis (1965).

It seems that to make a major contribution to science, talent and deep knowledge are necessary but not sufficient, and one also needs to be at the right place at the right time. It is fair to tell that V. A. Atsarkin was lucky that the 1960s saw the beginning of intense research on spin thermodynamics in solid electron and nuclear paramagnets, following a highly original work by Boris Provotorov who, essentially, created a modern formulation of the nuclear magnetic resonance (NMR) theory in solids. The Provotorov theory introduced the concept of the dipolar order characterizing mutual orientations of spins in local fields. Some experimental evidence for the applicability of this concept to NMR in solids was soon obtained, but the problem was still awaiting its final solution.

Naturally, the question of testing the Provotorov theory in EPR also presented itself. However, some experts were skeptical about the applicability to EPR of Provotorov's two-temperature picture because of a chaotic distribution of paramagnetic impurities in magnetically diluted paramagnetic crystals and inhomogeneous broadening of EPR lines. M. I. Rodak, a colleague of V. A. Atsarkin at the same laboratory, predicted that the two-temperature theory leads to a new effect: induced irradiation under saturation on the wing of the resonance line of the spin system. A series of original experiments carried out by V. A. Atsarkin (initially, with his student S. K. Morshnev) confirmed her prediction and brought a "smoking gun" for the applicability of the two-temperature theory to EPR. These pioneering studies of V. A. Atsarkin were among the earliest experimental confirmations of Provotorov's equations in all branches of magnetic resonance.

In subsequent investigations, V. A. Atsarkin with his colleague A. E. Mefed experimentally proved the existence of direct thermal contact between the electron dipole-dipole and nuclear Zeeman subsystems. This finding allowed them to discover an additional mechanism of nuclear spin relaxation via the electron dipolar reservoir and to implement a new mechanism of Dynamic Nuclear Polarization (DNP), the so-called "thermal mixing". This mechanism is widely used up to now along with such DNP methods as the Overhauser effect, the solid effect, and the cross-relaxation. These outstanding results served as the foundation of Atsarkin's Doctor of Science thesis defended in 1971.

In 1973, V. A. Atsarkin predicted theoretically and soon (together with O. A. Ryabushkin) proved experimentally the effect of enhanced longitudinal susceptibility. Performing measurements in the conditions of the EPR line's saturation, one can achieve the susceptibility enhancement by several orders ("the Atsarkin effect") due to the strong cooling of the electron dipolar reservoir. Further, V. A. Atsarkin with A. E. Mefed developed an original method of direct observation of NMR in rotating reference frames. The method yields a strong narrowing of the NMR line and allows the detection of ultra-slow molecular motion. 
Many influential contributions of V. A. Atsarkin involved applications of original new methods of EPR spectroscopy to solid-state physics. Among those contributions, we mention the investigation of the shape of the hole, burned out in the dipolar EPR spectrum, studies of electron spin-lattice relaxation in high-temperature superconductors and manganites, the analysis of the metal-insulator transition in fullerides, the problem of dipolar broadening and exchange narrowing of the EPR lines of paramagnetic centers on the surface of a solid, etc. Most of these studies were carried out with his colleagues and students, including V. V. Demidov, G. A. Vasneva, N. E. Noginova, and others. In recent years, V. A. Atsarkin has focused his efforts on research in the fields of ferromagnetic resonance and spin current in nanostructures; he has obtained some new results in studying the foundational problem of the transition from the quantum to classical behavior with the growth of the system size.

These impressive scientific achievements were accompanied by major contributions to the magnetic resonance community and the research infrastructure at the national and international level.

His broad scientific erudition, his deep knowledge, and his sincere desire to help colleagues understand difficult questions of magnetic resonance spectroscopy and spin dynamics explain the admiration and respect of the community to V. A. Atsarkin. Between conference talks, lines form by theorists and experimentalists who wish to discuss their work with Professor Atsarkin.

For 40 years, V. A. Atsarkin has led the seminar "Problems of magnetic resonance", which has become the central event for the Moscow magnetic resonance community. Numerous young scientists were deeply influenced by the seminar. Many outstanding results, presented at the seminar, received Atsarkin's support. Many participants, the speakers and the audience alike, developed a better understanding of the presented results.

Professor Atsarkin served as a long-time editor of Magnetic resonance in solids, he is a member of the Editorial Board of Applied Magnetic Resonance. For many years he led the Program Committee of the International School "Actual problems of magnetic resonance and its applications".

V. A. Atsarkin has devoted his life to science. He worked with infectious enthusiasms and never rested in the search of new ways to understand nature. Scientific depth and elegant logic in the work of V. A. Atsarkin are inseparable from his rare feeling of beauty and sublime. He is a gifted poet whose marvelous poems reflect this unique man's rich and unusual way of seeing the world.

One of many gifts of Vadim Atsarkin is his ability to cultivate intense human connections, as proved by the enthusiasm of the contributors to this Special Issue.

We hope that these papers, both original contributions and reviews written by friends and colleagues all around the world, will be of interest to many senior and junior scientists and will provide a glimpse at different fields touched upon by Vadim in his long career.

We heartily thank all the contributors and we are grateful to Professor Kev Salikhov and Dr. Laila Mosina for their support and encouragement during all the phases of the preparation of this special issue. In much the same way, our thanks go to the 
referees, who provided constructive criticisms thereby contributing to the improvement of the papers.

Edward B. Fel'dman, guest editor.

Publisher's Note Springer Nature remains neutral with regard to jurisdictional claims in published maps and institutional affiliations. 